\title{
Source time function clustering reveals patterns in earthquake dynamics
}

\author{
Jiuxun Yin ${ }^{1}$, Zefeng $\mathbf{L i}^{2}$, Marine Denolle ${ }^{1}$ \\ ${ }^{1}$ Department of Earth and Planetary Sciences, Harvard University, Cambridge, MA, USA \\ ${ }^{2}$ Seismological Laboratory, Division of Geological and Planetary Sciences, California Institute of \\ Technology, Pasadena, CA, USA
}

\section{Key Points:}

- We cluster earthquakes based on the dynamic time warping distance of their source time function (STF) shapes.

- The patterns of complexity correlate with source parameters such as depth, mechanism, and radiation.

- Simulations of dynamic rupture indicate a correlation between the STF complexity and frictional properties.

Corresponding author: Zefeng Li, zefengli@caltech.edu 


\begin{abstract}
We cluster a global data base of $3529 \mathrm{M}>5.5$ earthquakes in 1995-2018 based on a dynamic time warping dissimilarity of their source time functions (STFs). The clustering exhibits different degrees of STF shape complexity and suggests an association between STF complexity and earthquake source parameters. Thrust events are in large proportion with simple STF shapes and at all depths. In contrast, earthquakes with complex STF shapes tend to be located at shallow depth in complicated tectonic regions with preferentially strike slip mechanism and relatively longer duration. With 2D dynamic modeling of earthquake ruptures on heterogeneous pre-stress and linear slip-weakening friction, we find a systematic variation of the simulated STF complexity with frictional properties. Comparison between the observed and synthetic clustering distributions provides useful constraints on elements of the frictional properties. In particular, the characteristic slip-weakening distance could be constrained to be generally short $(<0.1 \mathrm{~m})$ and depth dependent.
\end{abstract}

\title{
Plain Language Summary
}

Seismic waves carry a signature about the earthquake source process. Earthquake source time functions (STFs), which are directly recovered from seismic waves, reflect the temporal history of earthquake rupture. However, it is often hard to directly compare STFs due to the large differences among earthquakes in terms of amplitude and duration. In this study, we perform a cluster analysis of STFs using a technique called dynamic time warping (DTW). DTW is commonly used in speech recognition to handle with various speeds of elocution. DTW allows us to dynamically stretch the seismic signals and provides a new way to quantify earthquake similarity through analyzing the shapes of their source time functions (STFs). We apply this to a large database of STFs. Our results show that the shape complexity of STFs is correlated with the earthquake source parameters such as the earthquake depth, focal mechanism, and energy radiation. Our numerical simulations further show that those correlations may indicate a spatial heterogeneity of frictional properties.

\section{Introduction}

Earthquakes are known to break in diverse manners: some events rupture on a geometrically simple fault with a relatively smooth slip distribution (e.g., Yagi \& Fukahata, 2011), while others break a network of faults and/or have heterogeneous slip distribution (Li et al., 1994; Ammon et al., 2005; Meng et al., 2012; Cesca et al., 2017). Although the complexity of earthquakes can be directly observed, in some cases, from surface fault trace (Massonnet et al., 1993; Li et al., 1994; Kaneko et al., 2017), many ruptures are buried at depth so that seismic waves are the only observations available to infer the source process. Derived from seismic waves through waveform deconvolution or kinematic inversion, the earthquake Source Time Function (STF) is a foremost important seismic observation that describes the time history of moment release during a rupture. Moreover, the shape of the STF directly controls the variability and uncertainty in the strength and duration of strong ground motion.

Observations of global earthquake STFs and source spectra have shown significant inter-event variability among earthquakes (Allmann \& Shearer, 2009; Atik et al., 2010; Denolle, 2019). Such variability may partly come from differences in data processing strategy (Ide \& Beroza, 2001). Therefore, large catalogs of STFs (or their spectra) obtained from a uniform approach is preferable to analyze relative differences among earthquakes (Allmann \& Shearer, 2009; Convers \& Newman, 2011; Denolle \& Shearer, 2016; Vallée \& Douet, 2016). 
Recently, such catalogs of STFs (or of their spectra) have enabled multiple discoveries about earthquake source processes. For example, the total seismic moment $M_{0}$ (the time integral of the STF) scales with source duration $T^{3}$ (the duration of the STF) for most small to moderate size earthquakes, which implies that the earthquake stress drop is roughly invariant with earthquake magnitudes. At larger magnitudes, this scaling may differ (e.g. $M_{0} \sim T^{2}$ from Denolle and Shearer (2016)). Their properties also have indicated that the ratio of the radiated energy $E_{R}$ over the moment, also referred to as the scaled energy $E_{R} / M_{0}$, varies spatially and with depth but remains invariant with earthquake magnitude (Convers \& Newman, 2011; Baltay et al., 2014; Denolle \& Shearer, 2016).

However, both the amplitude and the source duration of the STF vary by orders of magnitude. This requires careful strategies of amplitude and time scaling for acrossmagnitude visualization and comparison. One approach is to scale the time axis to a duration metric and normalize the amplitude to seismic moment (i.e. the integral of the STF). However, source duration is difficult to measure because near-source and near-site scattering of seismic waves may interfere with waves radiating from the end of the seismic rupture. Therefore previous studies have proposed several metrics of duration: momentbased duration (Houston, 2001), threshold-based duration (Vallée, 2013; Denolle, 2019), and centroid-based duration (Meier et al., 2017). Because these measures are not strictly equivalent, the shapes of the scaled and stretched STFs differ as well. For instance, Meier et al. (2017) find that average STFs have rather a triangle shape whereas Denolle (2019) suggests a rather skewed-Gaussian functional form.

Here, we propose to weaken the assumption of a particular definition of source duration and instead use dynamic time warping (DTW) to compare the shapes of the STFs. DTW has been widely used in speech recognition (Berndt \& Clifford, 1994; "Dynamic Time Warping", 2007). The DTW algorithm performs a non-uniform stretching of time and amplitude to match the shape of two time series via the optimal warping path with minimum distance (Figure S1). We measure the similarity between STFs with DTW distance and cluster the STFs accordingly. We apply this to the global SCARDEC cata$\log$ of STFs (Vallée \& Douet, 2016, available at http://scardec.projects.sismo.ipgp .fr/, last accessed 01/20/2020) that contains 3529 earthquakes of magnitude greater than 5.5 from $1 / 1 / 1992$ and until $12 / 31 / 2018$. The analysis shows that the STF overall shape is correlated with several earthquake source parameters, such as focal mechanisms, depth, and scaled energy.

To test whether the current physical understanding of earthquake processes reproduces the clustering patterns, we perform dynamic simulations of earthquake ruptures with linear slip-weakening friction to construct synthetic STFs. We find a strong correlation between the grouping distribution of STF shapes and frictional parameters, such as the characteristic slip-weakening distance $D_{c}$. Furthermore, we find that the grouping pattern of the SCARDEC STF shapes are most similar to those simulated STFs with small values of $D_{c}$, thus the grouping patterns of a large number of STFs can potentially provide observational constraints to earthquake dynamics.

\section{Dynamic time warping and clustering analysis}

DTW measures the similarity between two time series that may not share the same frequency content or the same sampling rate. The series are "warped" (or stretched) nonuniformly in the time dimensions to optimally match two series (Figure S1). This algorithm is widely used in automated speech recognition in which different audio sequences may have different speaking speeds (Berndt \& Clifford, 1994; "Dynamic Time Warping", 2007). One important advantage of DTW is its ability to preserve topological structures of the time series by assimilating their temporal elongation or compression. Once stretched, the DTW distance is taken as a new metric for STF similarity, which can be used for 
clustering. Our approach follows four steps: 1) STF pre-conditioning, 2) DTW distance calculation, 3) clustering, 4) re-grouping around a centroid event.

We first perform minimal pre-conditioning of the STF shapes. The STFs are built from the deconvolution of teleiseismic $\mathrm{P}$ waves that are relatively well constrained at frequencies below $1 \mathrm{~Hz}$ (Vallée \& Douet, 2016). Given that the maximum duration of the STF in the catalog is about $100 \mathrm{~s}$, we re-sample the data to 100 points giving a minimum sampling rate of 1 point per second. We then normalize the amplitude STFs to the event seismic moment. These two processing steps improve the stability of the warping. We have tested various strategies to resample and normalize the STFs, which did not affect the conclusions of this analysis.

Second, we apply the DTW to each pair of STFs. The DTW distance is the Euclidean distance between two STFs warped along the optimal warping path, and is chosen here as the measure of similarity between two STFs (see Figure S1 (a)-(b)).

Then, the STF shapes are clustered based on their DTW distance with a singlelinkage hierarchical clustering analysis that provides the flexibility to form clusters at any desired level (Text S1, Figure S1 (c)). Here, we constrain the number of clusters to be 20, which is about equivalent of DTW distance threshold of 0.4. For each of these clusters, we choose a representative STF (defined as the centroid event) that has the minimum median distance with all of the other members of the cluster. It is similar to the stack of all stretched STF within each cluster (Figure 1), which, in turn, exhibits the common features of all cluster members.

Furthermore, we parameterize the characteristic STF shape for each of these clusters by calculating the number of prominent peaks of each centroid event. The number of prominent peaks is commonly used for topographic relief analysis and is defined as the amplitude of the peak (hill summit) relative to the lowest amplitude point (valley) that does not contain a higher peak. This metric differs from the calculation of Gaussian subevents that Danré et al. (2019) use. One hyper-parameter we tune is a threshold for peak amplitude of the prominent peak, which we choose to be $10 \%$ of the global maximum of the STF amplitude. The raw and stretched STFs have a lot fewer prominent peaks than individual peaks from the Gaussian decomposition by Danré et al. (2019) (Figure S2). Furthermore, the stretched STFs have fewer prominent peaks than the raw STFs, but in general the same number of prominent peaks as the centroid event (Figure S3). For instance, a STF may have multiple separated amplitude peaks, but only one single prominent peak (Figure 1 (a)-(b)).

Finally, we group the clusters based on the number of prominent peaks of the centroid event, where G1 is the group where the centroid event has 1 prominent peak, G2 is the group where the centroid event has 2 prominent peaks, ... (Figure 1 (c)). G4 is the group where the centroid event has at least 4 prominent peaks. Examples of detected prominent peaks are found in Figure 1 (a)-(b) (see Figure S4 for the unstretched STFs). In this study, we define the STFs to be "complex" if their DTW stretched STFs have multiple prominent peak. The first order result from the grouping is that most events have a single prominent peak whereas about $20 \%$ events are more complex.

\section{Correlations between shape complexity and source parameters}

We now explore the correlation between grouping and several source parameters such as depth, focal mechanism, moment, duration, energy, and location.

The first property we investigate is the source depth. Complex STFs (groups G2G4) are mostly shallow crustal events $(\leq 20 \mathrm{~km})$ whereas the simple STFs (group G1) can be found at all depths (Figure $2(\mathrm{a})$ ). Because co-located events have various degrees 


\section{(a) SCARDEC STFs}

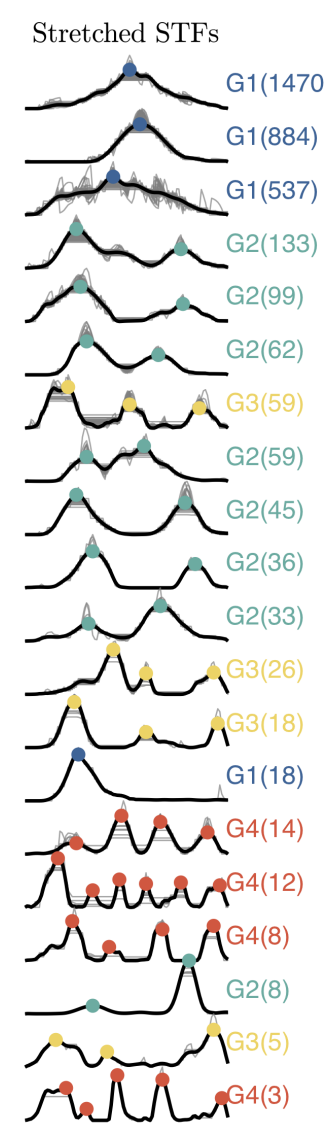

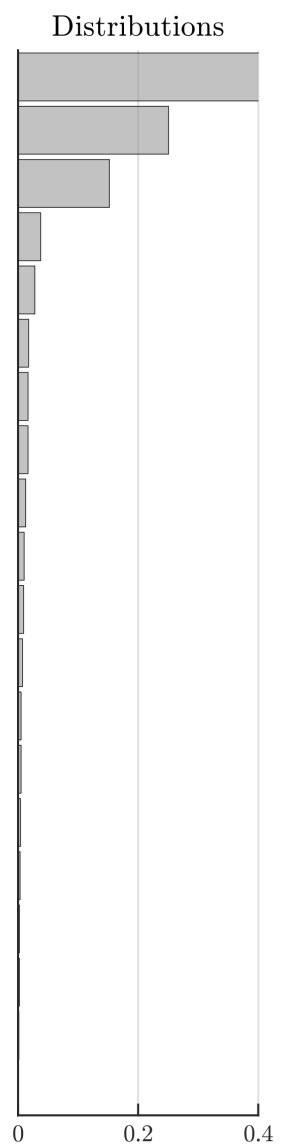

(b) Simulated STFs $(\mathrm{Dc}=0.1 \mathrm{~m})$
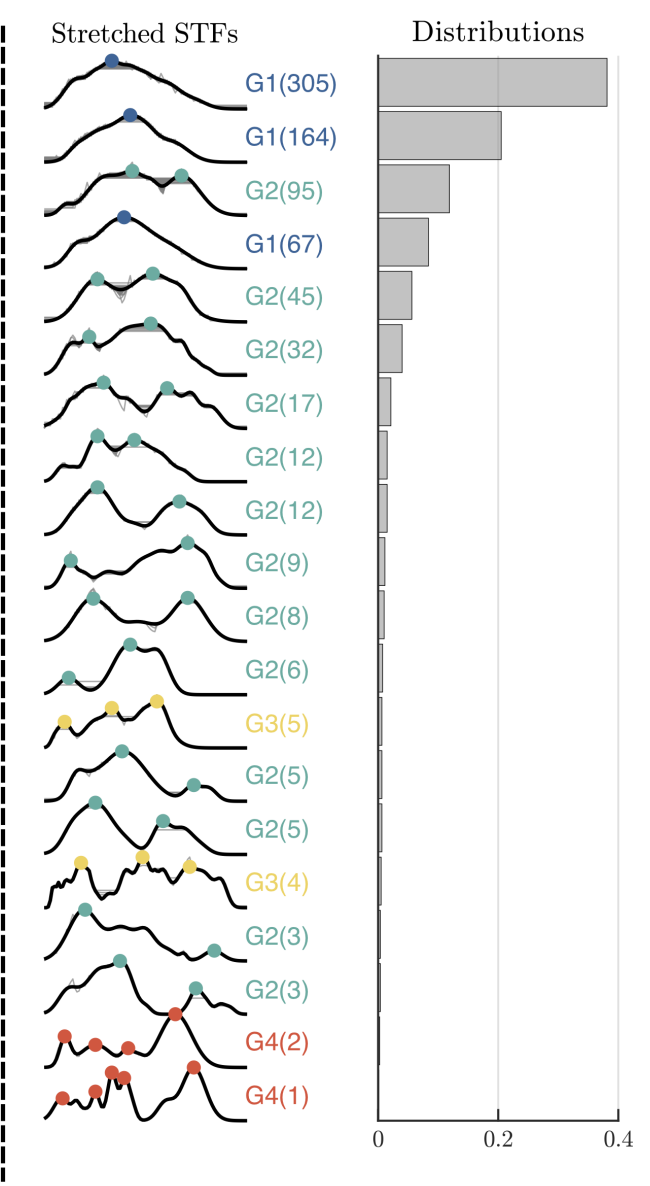

(c)
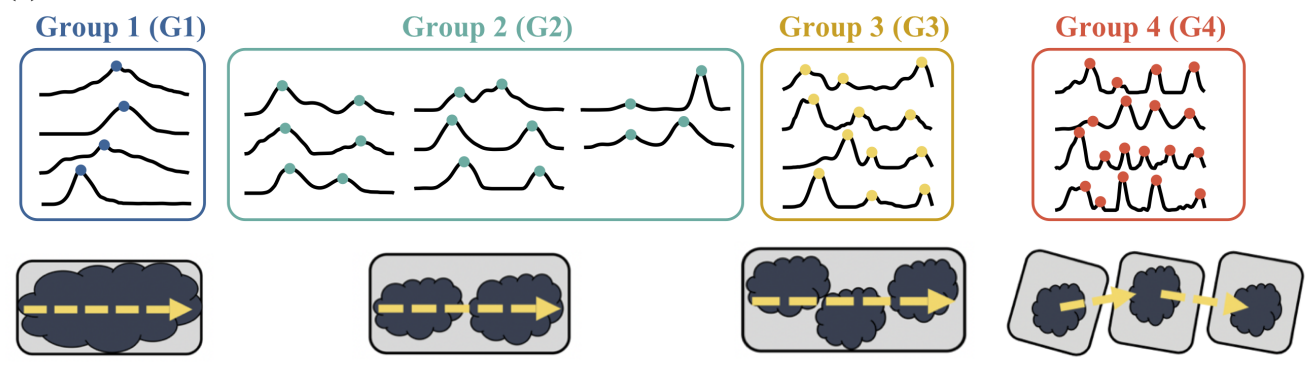

Figure 1. Source time function clustering, grouping, and conceptual interpretation. (a) Individual STFs after dynamic time warping and clustering are shown by gray thin lines. Black thick lines are the STFs of the centroid event of each cluster. Colored dots indicate the prominent peaks of the centroid STF as well as the associated group. Numbers in the parentheses are the number of STFs in each cluster. The corresponding population proportion of each cluster is shown in the right histograms. (b) Same as (a) but for the STFs from our dynamic simulations. (c) Cluster centroid STF shapes and conceptual models for G1-G4. In the model diagram, dark blocks represent major rupture asperities and the arrow indicates the rupture direction. bias these specific results. 

Figure 6). Therefore, we propose that the complexity in the STF may reflect the complexity in the regional stress field.

\section{Modeling STF complexity}

Simulations of dynamic ruptures using stochastic distributions of fault-interface parameters are popular in the investigations of complex kinematic source models, realistic fault geometry and roughness models, and to simulate high-frequency ground motions (Mai \& Beroza, 2002; Ripperger et al., 2007; Trugman \& Dunham, 2014; Graves \& Pitarka, 2016; Mai et al., 2017). In order to investigate possible factors that control the STF complexity patterns, we perform a large number of 2-dimensional dynamic rupture simulations with stochastic distributions of pre-stress, and apply the same clustering analysis to the resulting synthetic STFs as to the SCARDEC STFs.

In this study, synthetic dynamic sources are generated in a 2-dimensional medium in an anti-plane setting. Pre-stress on the fault is constrained to follow a power-law amplitude distribution that approximates the scenario caused by natural fault roughness (Candela et al., 2012, Text S2 for more details). We assume a constant normal stress of $120 \mathrm{MPa}$ and linear slip weakening friction law (Andrews, 1976). Linear slip weakening requires three parameters: the static friction coefficient (here chosen as $\mu_{s}=0.677$ ), the dynamic friction coefficient (here chosen as $\mu_{d}=0.525$ ), and the characteristic slipweakening distance $D_{c}$. We set up the experiments so that the fault-average stress drop is about $1 \mathrm{MPa}$ (Figure S7).

Danré et al. (2019) find that heterogeneity is necessary to reproduce realistically rough STFs. Here, we focus on varying $D_{c}$, yet aware of the trade-off between strength excess and $D_{c}$ in controlling rupture velocity and the resulting ground motions (Guatteri \& Spudich, 2000). While we keep $D_{c}$ constant within a single set of simulations, we carry several sets of experiments with values of $D_{c}$ at various levels $0.05,0.1,0.2,0.4,0.8$, and $1.6 \mathrm{~m}$ that are within bounds found in the literature.

For each $D_{c}$, we first generate a set of pre-stress distributions that we use in each simulations. The dynamic rupture is solved by $2 \mathrm{D}$ boundary integral method SBIEMLAB ( http://web.gps.caltech. edu/ ampuero/sof tware.html, last accessed 11/27/2018). We discard the rupture models that unsuccessfully nucleated with a source dimension less than $20 \mathrm{~km}$, or rupture beyond the zone of heterogeneous pre-stress, and obtain 800 qualified simulations for each $D_{c}$ value. Finally, the STFs are calculated from the integral of the moment-density-rate functions over the fault surface (more details in Text S2).

We perform the hierarchical clustering and group the simulated STFs for each $D_{c}$, following the same procedures as for the SCARDEC STFs (Figure 1 (b), Figures S8 S12). Because our modeling is not three dimensional and does not include the free surface, we are not matching observations such as the focal mechanism and depth. However, our results can match the proportion of the STFs relative to each group: $80 \%$ of the STFs belong to the G1 group, 15\% belong to the G2, and the rest in higher indexed groups. Comparison of the relative proportion between groups for each set of simulations suggests that an increasing $D_{c}$ value yield an increase in STF complexity (e.g. proportion of G3-G4 events). This shows that $D_{c}$, or more generally, the frictional parameters can impact the complexity of STFs. Compared with the observed global variability in SCARDEC STFs, small value of $D_{c}(<0.1 \mathrm{~m})$ is preferred in this particular metric of complexity. In contrast, models with large value of $D_{c}$ tend to generate proportionally more STFs belonging to G3 beyond (Figures S10 - S12).

Our results indicate that the small values of $D_{c}<0.1 \mathrm{~m}$ are necessary to produce the general level of complexity of the SCARDEC STFs (Figure 5 (a)). When binning these relative contributions with source depths, we find that crustal events ( $\mathrm{h} \leq 40 \mathrm{~km})$, 

software.html). The Matlab scripts to reproduce the results and figures can be obtained on the Github (https://github.com/yinjiuxun/STF_DTW). Global maps are made by GMT (Wessel et al., 2013, available at http://gmt.soest.hawaii.edu/) ).

\section{References}

Allmann, B. P., \& Shearer, P. M. (2009). Global variations of stress drop for moderate to large earthquakes. Journal of Geophysical Research: Solid Earth, 114(B1), B01310. doi: 10.1029/2008JB005821

Ammon, C. J., Ji, C., Thio, H.-K., Robinson, D., Ni, S., Hjorleifsdottir, V., ... Wald, D. (2005). Rupture Process of the 2004 Sumatra-Andaman Earthquake. Science, 308(5725), 1133-1139. doi: 10.1126/science.1112260

Andrews, D. J. (1976). Rupture propagation with finite stress in antiplane strain. Journal of Geophysical Research, 81(20), 3575-3582. doi: 10.1029/ JB081i020p03575

Atik, L. A., Abrahamson, N., Bommer, J. J., Scherbaum, F., Cotton, F., \& Kuehn, N. (2010). The Variability of Ground-Motion Prediction Models and Its Components. Seismological Research Letters, 81(5), 794-801. doi: 10.1785/gssrl.81.5.794

Baltay, A. S., Beroza, G. C., \& Ide, S. (2014). Radiated Energy of Great Earthquakes from Teleseismic Empirical Green's Function Deconvolution. Pure and Applied Geophysics, 171(10), 2841-2862. doi: 10.1007/s00024-014-0804-0

Berndt, D. J., \& Clifford, J. (1994). Using dynamic time warping to find patterns in time series. In KDD workshop (Vol. 10, pp. 359-370). Seattle, WA.

Bird, P. (2003). An updated digital model of plate boundaries. Geochemistry, Geophysics, Geosystems, 4(3). doi: 10.1029/2001GC000252

Brune, J. N. (1971). Correction (to Brune, 1970). J. geophys. Res, 76, 5002.

Candela, T., Renard, F., Klinger, Y., Mair, K., Schmittbuhl, J., \& Brodsky, E. E. (2012). Roughness of fault surfaces over nine decades of length scales. Journal of Geophysical Research: Solid Earth, 117(B8). doi: 10.1029/2011JB009041

Cesca, S., Zhang, Y., Mouslopoulou, V., Wang, R., Saul, J., Savage, M., ... Dahm, T. (2017). Complex rupture process of the Mw 7.8, 2016, Kaikoura earthquake, New Zealand, and its aftershock sequence. Earth and Planetary Science Letters, 478, 110-120. doi: 10.1016/j.epsl.2017.08.024

Convers, J. A., \& Newman, A. V. (2011). Global evaluation of large earthquake energy from 1997 through mid-2010. J. Geophys. Res. Solid Earth. doi: 10.1029/ 2010JB007928

Danré, P., Yin, J., Lipovsky, B. P., \& Denolle, M. A. (2019). Earthquakes Within Earthquakes: Patterns in Rupture Complexity. Geophysical Research Letters, 46 (13), 7352-7360. doi: 10.1029/2019GL083093

Denolle, M. A. (2019). Energetic Onset of Earthquakes. Geophysical Research Letters , 46(5), 2458-2466. doi: 10.1029/2018GL080687

Denolle, M. A., \& Shearer, P. M. (2016). New perspectives on self-similarity for shallow thrust earthquakes. Journal of Geophysical Research: Solid Earth, 121(9), 2016JB013105. doi: 10.1002/2016JB013105

Dynamic Time Warping. (2007). In M. Müller (Ed.), Information Retrieval for Music and Motion (pp. 69-84). Berlin, Heidelberg: Springer. doi: 10.1007/978-3 $-540-74048-3 \_4$

Dziewonski, A. M., \& Anderson, D. L. (1981). Preliminary reference Earth model. Physics of the Earth and Planetary Interiors, 25(4), 297-356. doi: 10.1016/0031-9201(81)90046-7

Eshelby, J. D. (1957). The determination of the elastic field of an ellipsoidal inclusion, and related problems. In Proceedings of the Royal Society of London A: Mathematical, Physical and Engineering Sciences (Vol. 241, pp. 376-396). The Royal Society. 
Graves, R., \& Pitarka, A. (2016). Kinematic Ground-Motion Simulations on Rough Faults Including Effects of 3d Stochastic Velocity PerturbationsKinematic Ground-Motion Simulations on Rough Faults. Bulletin of the Seismological Society of America, 106 (5), 2136-2153. doi: 10.1785/0120160088

Guatteri, M., \& Spudich, P. (2000). What Can Strong-Motion Data Tell Us about Slip-Weakening Fault-Friction Laws? Bulletin of the Seismological Society of America, 90(1), 98-116. doi: 10.1785/0119990053

Houston, H. (2001). Influence of depth, focal mechanism, and tectonic setting on the shape and duration of earthquake source time functions. Journal of Geophysical Research: Solid Earth, 106(B6), 11137-11150. doi: 10.1029/2000JB900468

Ide, S., \& Beroza, G. C. (2001). Does apparent stress vary with earthquake size. Geophys. Res. Lett, 28(17), 3349-3352.

Ide, S., \& Takeo, M. (1997). Determination of constitutive relations of fault slip based on seismic wave analysis. Journal of Geophysical Research: Solid Earth, 102(B12), 27379-27391. doi: 10.1029/97JB02675

Kanamori, H., Hong-Kie, T., Doug, D., Egill, H., \& Heaton, T. (1992). Initial investigation of the Landers, California, Earthquake of 28 June 1992 using TERRAscope. Geophysical Research Letters, 19(22), 2267-2270. doi: 10.1029/92GL02320

Kaneko, Y., Fukuyama, E., \& Hamling, I. J. (2017). Slip-weakening distance and energy budget inferred from near-fault ground deformation during the 2016 Mw7.8 Kaikōura earthquake. Geophysical Research Letters, 44(10), 4765-4773. doi: $10.1002 / 2017$ GL073681

Kaneko, Y., \& Lapusta, N. (2010). Supershear transition due to a free surface in 3 -D simulations of spontaneous dynamic rupture on vertical strike-slip faults. Tectonophysics, 493(3), 272-284. doi: 10.1016/j.tecto.2010.06.015

Klinger, Y. (2010). Relation between continental strike-slip earthquake segmentation and thickness of the crust. Journal of Geophysical Research: Solid Earth, 115(B7). doi: 10.1029/2009JB006550

Li, Y.-G., Aki, K., Vidale, J. E., Lee, W. H. K., \& Marone, C. J. (1994). Fine Structure of the Landers Fault Zone: Segmentation and the Rupture Process. Science, 265(5170), 367-370. doi: 10.1126/science.265.5170.367

Mai, P. M., \& Beroza, G. C. (2002). A spatial random field model to characterize complexity in earthquake slip. Journal of Geophysical Research: Solid Earth, 107(B11), ESE 10-1. doi: 10.1029/2001JB000588

Mai, P. M., Galis, M., Thingbaijam, K. K. S., Vyas, J. C., \& Dunham, E. M. (2017). Accounting for Fault Roughness in Pseudo-Dynamic GroundMotion Simulations. Pure and Applied Geophysics, 174(9), 3419-3450. doi: 10.1007/s00024-017-1536-8

Massonnet, D., Rossi, M., Carmona, C., Adragna, F., Peltzer, G., Feigl, K., \& Rabaute, T. (1993). The displacement field of the Landers earthquake mapped by radar interferometry. Nature, 364(6433), 138-142. doi: 10.1038/364138a0

Meier, M.-A., Ampuero, J. P., \& Heaton, T. H. (2017). The hidden simplicity of subduction megathrust earthquakes. Science, 357(6357), 1277-1281. doi: 10 $.1126 /$ science.aan5643

Meng, L., Ampuero, J.-P., Stock, J., Duputel, Z., Luo, Y., \& Tsai, V. C. $\quad$ (2012). Earthquake in a maze: Compressional rupture branching during the $2012 \mathrm{Mw}$ 8.6 Sumatra earthquake. Science, 337(6095), 724-726.

Peyrat, S., Olsen, K., \& Madariaga, R. $\quad$ (2001, November). Dynamic modeling of the 1992 Landers earthquake. Journal of Geophysical Research: Solid Earth, 106 (B11), 26467-26482. doi: 10.1029/2001JB000205

Ripperger, J., Ampuero, J.-P., Mai, P. M., \& Giardini, D. (2007). Earthquake source characteristics from dynamic rupture with constrained stochastic fault stress. Journal of Geophysical Research: Solid Earth, 112(B4). doi: 
10.1029/2006JB004515

Shearer, P. M., Prieto, G. A., \& Hauksson, E. (2006). Comprehensive analysis of earthquake source spectra in southern California. Journal of Geophysical Research: Solid Earth, 111 (B6). doi: 10.1029/2005JB003979

Trugman, D. T., \& Dunham, E. M. (2014). A 2d Pseudodynamic Rupture Model Generator for Earthquakes on Geometrically Complex FaultsA 2d Pseudodynamic Rupture Model Generator for Earthquakes on Geometrically Complex Faults. Bulletin of the Seismological Society of America, 104(1), 95-112. doi: $10.1785 / 0120130138$

Vallée, M. (2013). Source time function properties indicate a strain drop independent of earthquake depth and magnitude. $\quad$ Nature Communications, 4, 2606. doi: 10.1038/ncomms3606

Vallée, M., Charléty, J., Ferreira, A. M. G., Delouis, B., \& Vergoz, J. SCARDEC: a new technique for the rapid determination of seismic moment magnitude, focal mechanism and source time functions for large earthquakes using body-wave deconvolution. Geophysical Journal International, 184(1), 338-358. doi: 10.1111/j.1365-246X.2010.04836.x

Vallée, M., \& Douet, V. (2016). A new database of source time functions (STFs) extracted from the SCARDEC method. Physics of the Earth and Planetary Interiors, 257, 149-157. doi: 10.1016/j.pepi.2016.05.012

Wessel, P., Smith, W. H. F., Scharroo, R., Luis, J., \& Wobbe, F. (2013). Generic Mapping Tools: Improved Version Released. Eos, Transactions American Geophysical Union, 94(45), 409-410. doi: 10.1002/2013EO450001

Wibberley, C. A. J., \& Shimamoto, T. (2005). Earthquake slip weakening and asperities explained by thermal pressurization. Nature, 436(7051), 689-692. doi: 10 $.1038 /$ nature03901

Yagi, Y., \& Fukahata, Y. (2011). Rupture process of the 2011 Tohoku-oki earthquake and absolute elastic strain release. Geophysical Research Letters, 38(19). doi: $10.1029 / 2011$ GL048701 
(a)

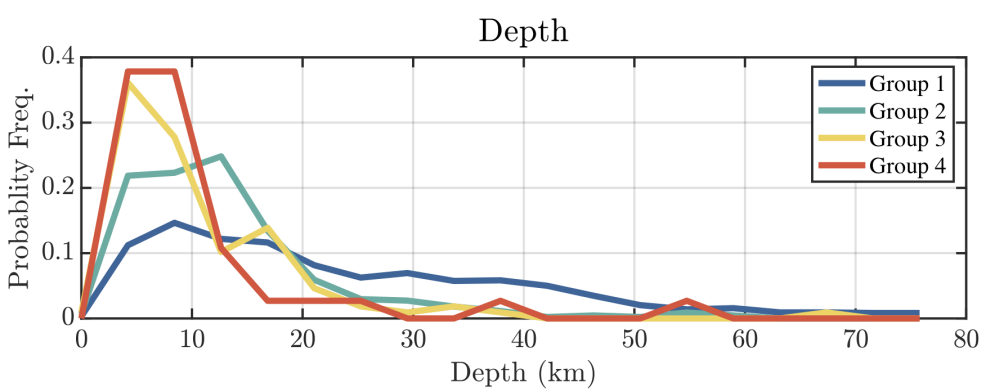

(b)

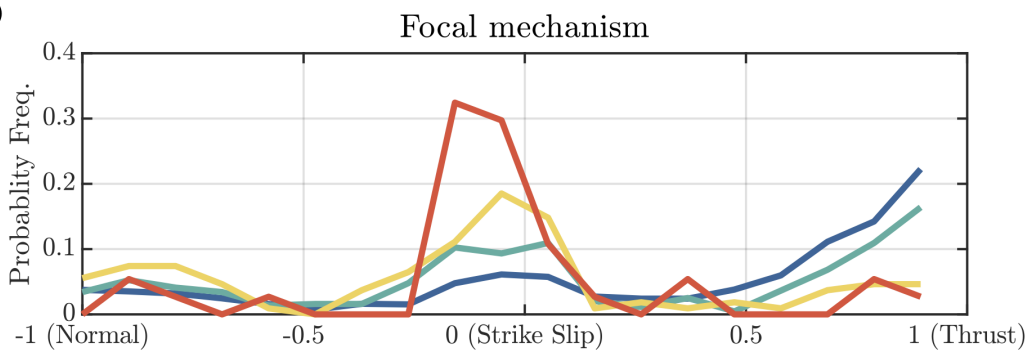

(c)

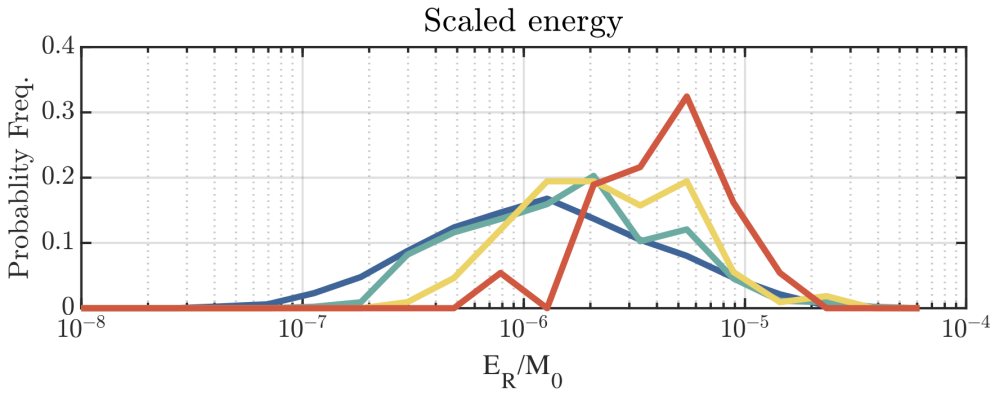

(d)

SCARDEC

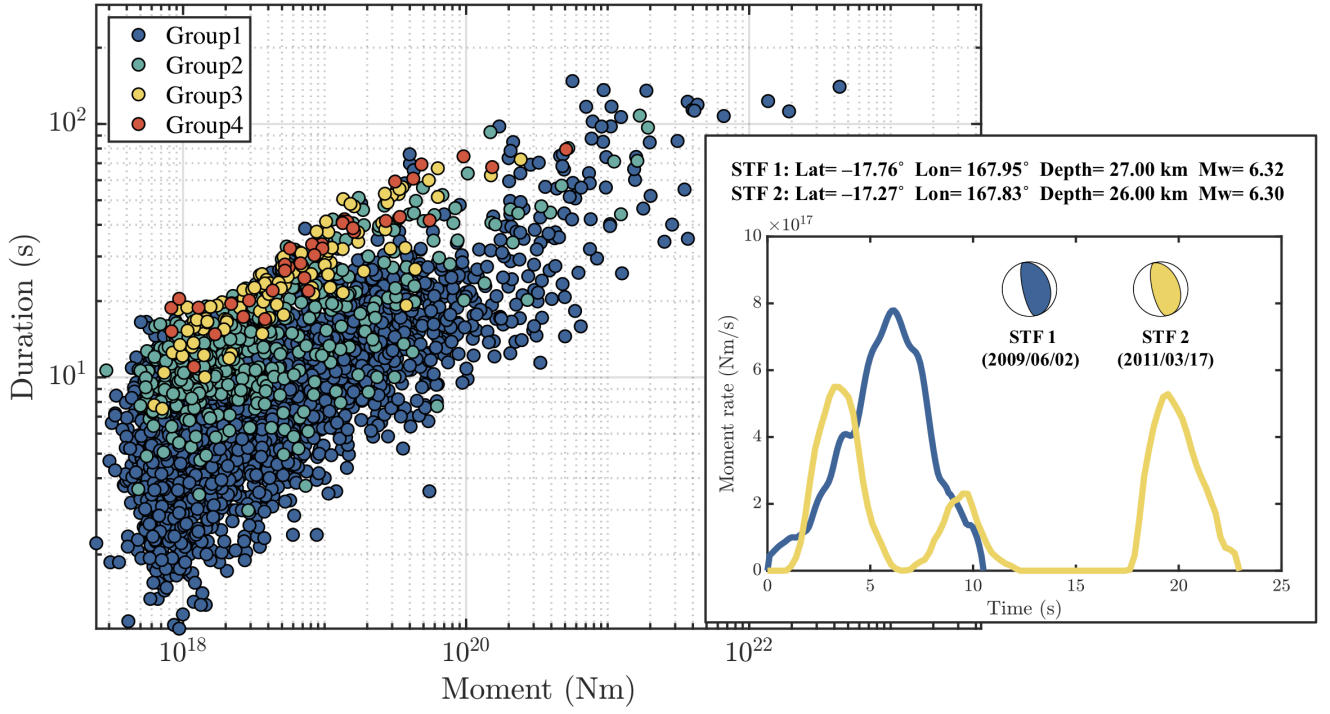

Figure 2. Population distribution of four complexity groups and correlation with different source parameters: (a) centroid depth, (b) focal mechanism (scalar defined by Shearer et al. (2006) that varies from -1 (normal), 0 (strike-slip) to 1 (reverse)), (c) and scaled radiated energy $e=E_{R} / M_{0}$. Panel (d) shows the earthquake duration against earthquake moment, colored with the respective group labels. One pair of co-located events with different complexity are also shown in the inset. 


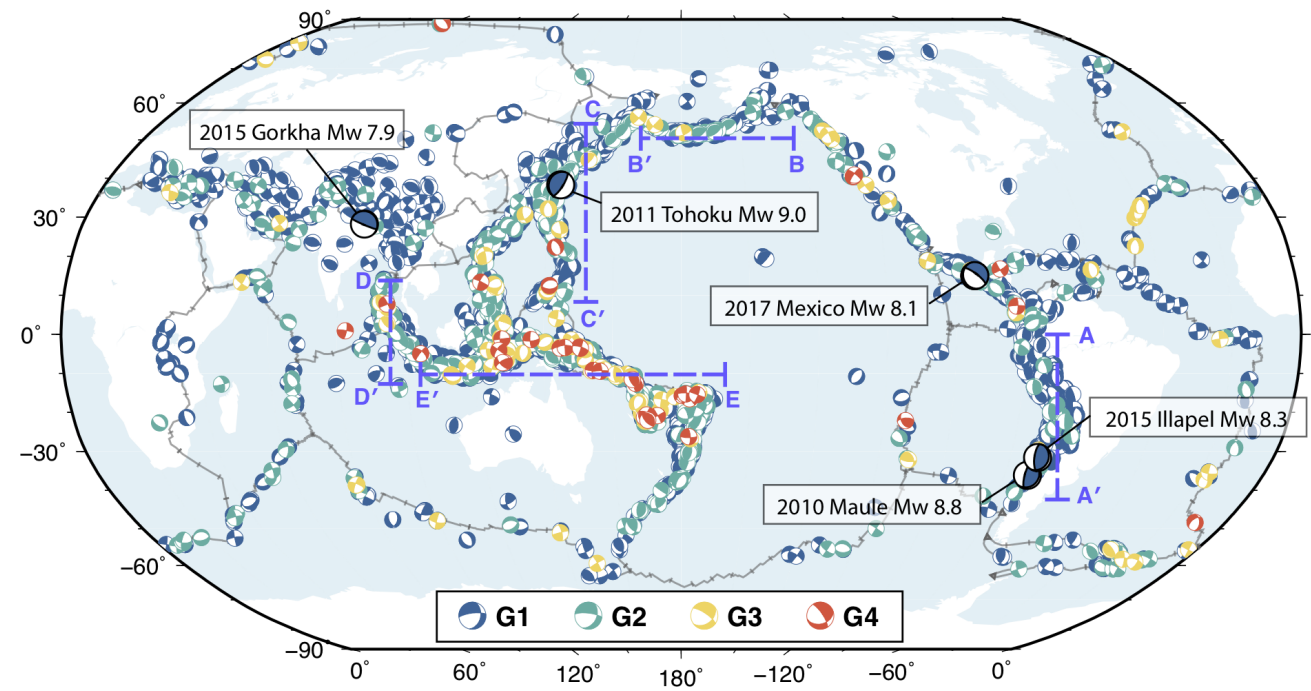

Figure 3. Map of focal mechanisms colored by their group label and overlay of the plate boundaries (gray thin lines). Several recent large megathrust earthquakes are highlighted. Blue dashed lines shown the locations of profiles in Figure 4. Bottom panels show the center STFs in each groups (same as those in Figure 1 (a)) as well as the corresponding schematic rupture propagation. 


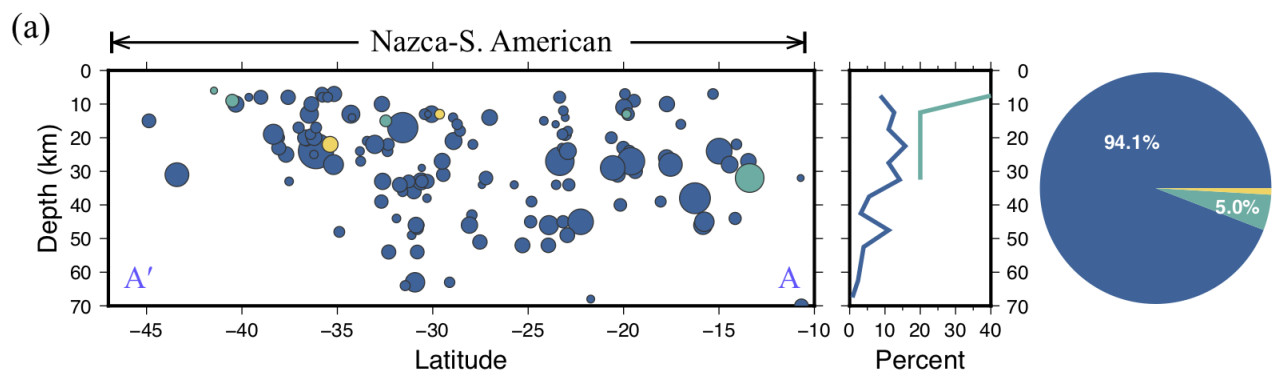

(b) $\longleftarrow$ Aleutian Islands $\longrightarrow$ Alaska $\longrightarrow$ 1
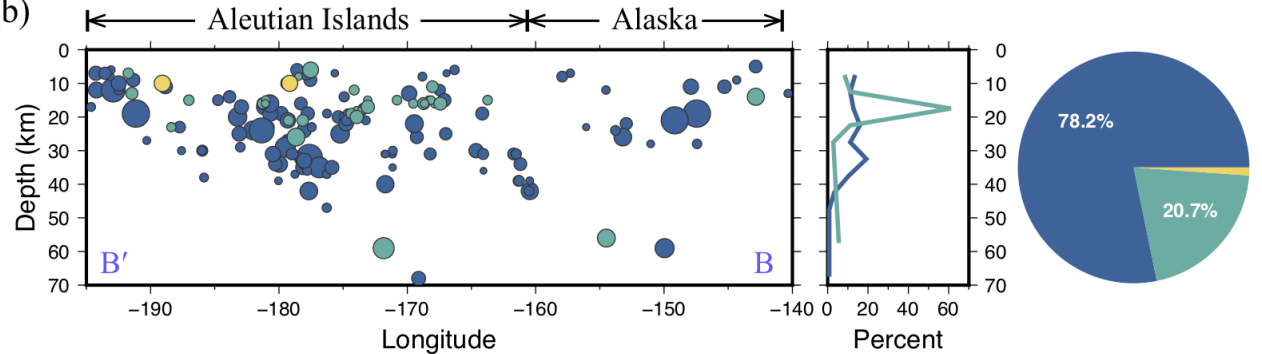

(c) $\quad k$ Mariana $*$ Izu-Bonin $\rightarrow$ Japan $\rightarrow$ Kuril $\rightarrow 1$

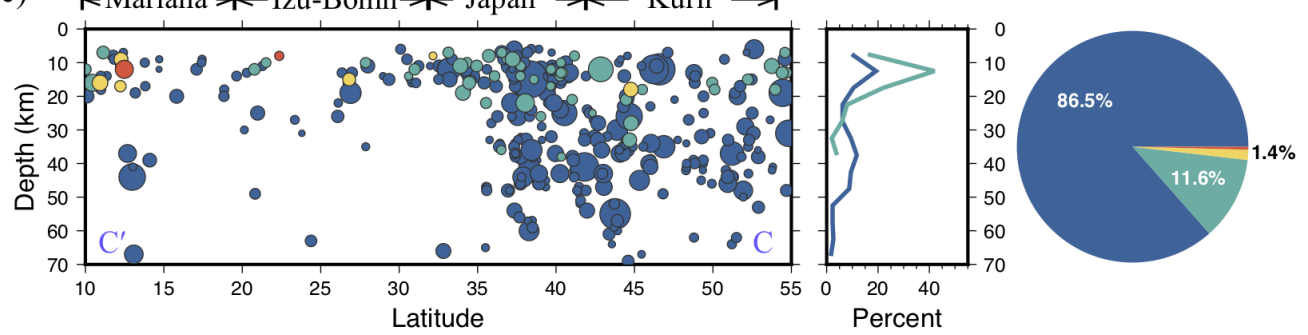

(d) $\longleftarrow$ Sunda trench $\longrightarrow$ Indian-Burma $\longrightarrow$ -

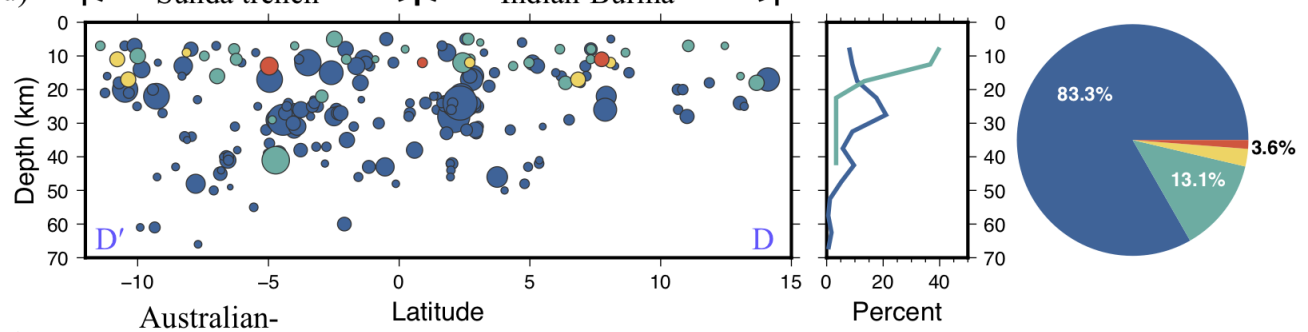

(e) $\quad \leftarrow$ Eurasian $\rightarrow \longleftarrow$ Australian-Pacific $\longrightarrow$ I

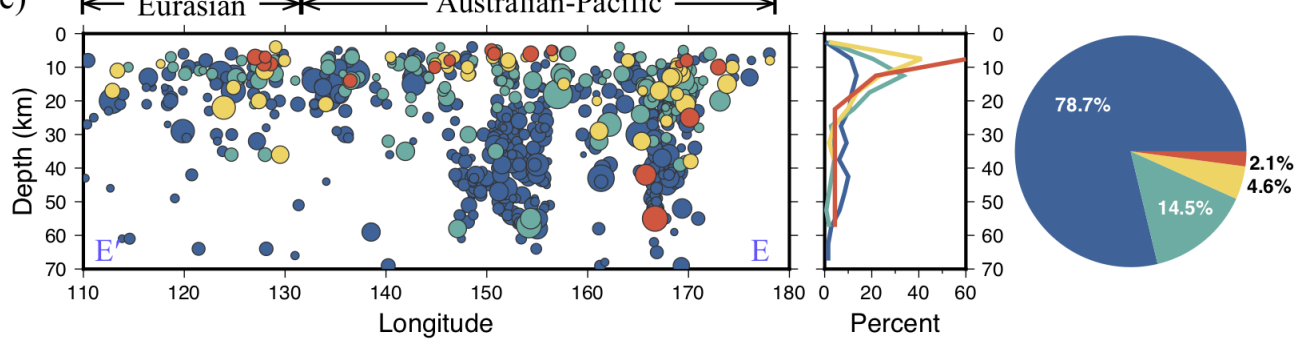

Figure 4. Earthquake distributions of different complexity groups on the vertical profiles (from 0-70 km, locations are indicated by blue dashed lines in Figure 3). The regional alongdepth and total group distributions are also shown to the right. 
(a)

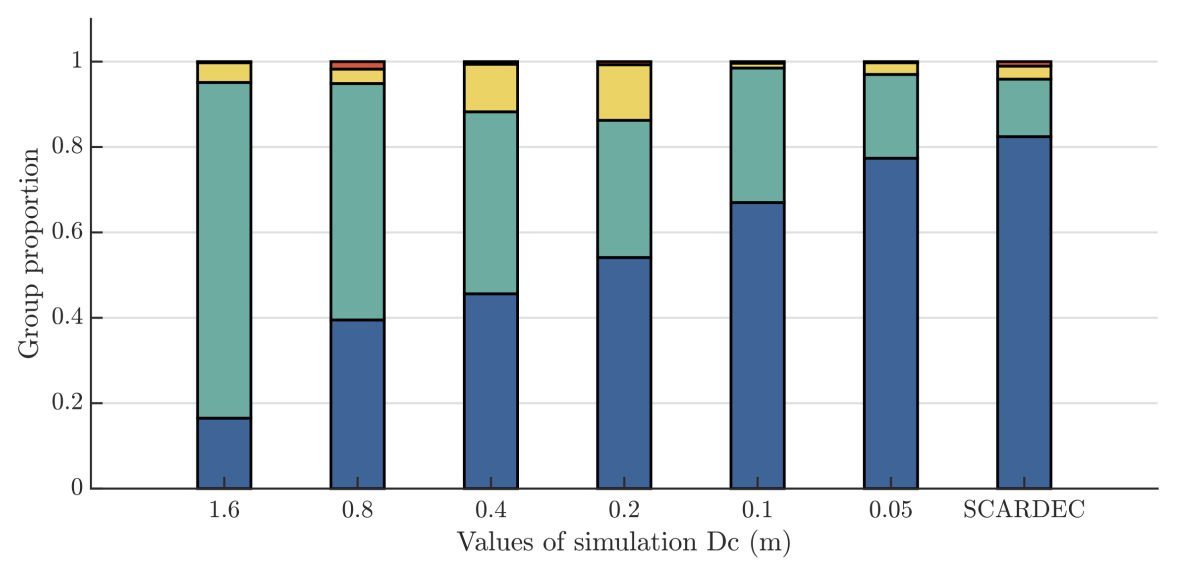

(b)

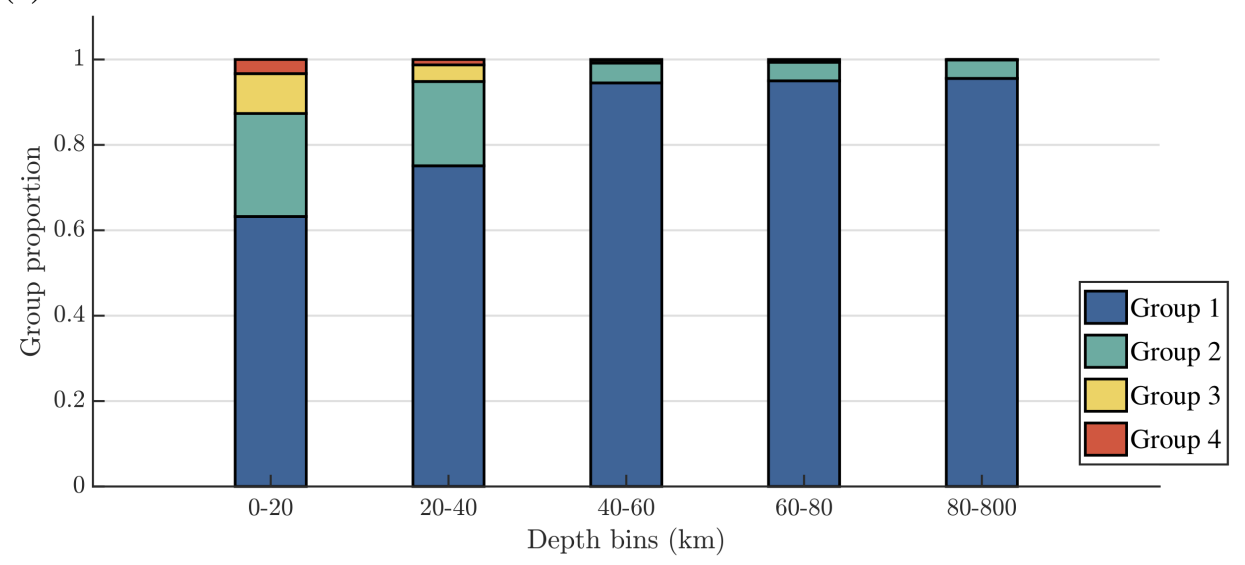

Figure 5. Group proportion distributions: (a) simulated STFs clustering with different values of $D_{c}$, compared with the group proportions of real STFs (SCARDEC); (b) Group proportions of real STFs (SCARDEC) within different depth bins. 